\title{
Relación del diseño de las botas de fútbol con la lesión en el fútbol: revisión sistemática
}

\author{
Relationship of the design of football boots to the injury in football: systematic review \\ Tatiana Rodríguez Macías y Eva Lopezosa Reca \\ Departamento de Podología. Universidad de Málaga, España
}

Palabras clave:

Fútbol, lesiones, botas de fútbol.

Keywords:

Soccer, injuries, boots, shoes.

\section{Resumen}

Objetivos: El fútbol es uno de los deportes más populares del mundo, con una alta incidencia de lesiones, principalmente en rodilla y tobillo. El propósito de este estudio fue recopilar información de la literatura disponible hasta el momento sobre la relación del diseño de las botas de fútbol con las lesiones en el fútbol.

Material y métodos: La búsqueda de la literatura se ha llevado a cabo en las bases de datos de PubMed, Scopus y Cinahl. Los términos utilizados fueron: "soccer", "football", "injuries", "boots" y "shoes" por separado y con los términos booleanos "OR" y "\&". La búsqueda se llevó a cabo entre enero y abril de 2020. El diseño de esta revisión sigue el método PRISMA y la de calidad de evidencia científica se ha evaluado a través de CASPe.

Resultados: La lesión en el fútbol es resultado de un patrón multifactorial, entre factores intrínsecos y extrínsecos.

Conclusión: La configuración de los tacos de fútbol está diseñada en función de la capacidad de penetración y amortiguación del terreno para los que están indicados. Las diferentes configuraciones de tacos pueden estar relacionadas con diferentes lesiones que se producen en el fútbol, en constante interacción con factores intrínsecos y extrínsecos.

\section{Abstract}

Objectives: Soccer is one of the most popular sports in the world, in which there is a high incidence of injuries, mainly on knees and ankles. The purpose of this study was to collect information from the literature available until present about the relationship between the design of the soccer boots and the soccer injuries.

Material and methods: The search of the literature was conducted through the different databases- Pubmed, Scopus and Cinahl. The terms used were: "soccer", "football", "injuries", "boots" and "shoes". The search was carried out between January and April 2020. The design of this review follows the PRISMA method and the quality of the scientific evidence has been evaluated through CASPe.

Results: Soccer injuries are the result of a multifactorial pattern between intrinsic and extrinsic factors.

Conclusion: The configuration of the soccer cleats is configured based on the penetration capacity and shock-absorption of the terrain for which they are intended. The different configurations of cleats can be related to different injuries that practicing soccer generates, in a constant interaction within intrinsic and extrinsic factors. 


\section{INTRODUCCIÓN}

El fútbol español sigue creciendo. Casi un millón de licencias fueron expedidas en la temporada 2015-16, manteniendo la tendencia al alza de las últimas temporadas. En total fueron 923.805 las licencias emitidas, creciendo en 72.501 futbolistas ${ }^{1}$.

Un factor importante del equipamiento que puede influir en unas mayores fuerzas de reacción del suelo en el fútbol es la bota ${ }^{2}$. Correr o realizar cambios repentinos en la dirección incrementa y al mismo tiempo influye en la dirección de las fuerzas que actúan sobre los miembros inferiores. Esto puede derivar en lesiones por sobreuso (o sin contacto) del aparato locomotor. El agarre proporcionado por diseño del taco podría influir de manera importante en estas fuerzas ${ }^{2,3}$.

Desde la implantación del césped artificial, este tipo de superficies han ido evolucionando hasta la llamada tercera generación. Por otro lado, el desarrollo de calzado específico para la práctica de fútbol sobre césped artificial no ha ido a la par con el desarrollo de dichas superficies. Esto afecta a una serie de aspectos de la interacción entre los usuarios y la superficie como el confort térmico, la absorción de impactos o la tracción, lo que se traduce en un bajo rendimiento deportivo y en la aparición de problemas como una mayor incidencia de lesiones de miembro inferior ${ }^{4,5}$.

\section{Botas de fútbol}

Las botas de fútbol han cambiado considerablemente en los últimos años. Uno de los principales cambios ha sido el peso ${ }^{2,3}$. Desde los más de $500 \mathrm{~g}$ de las primeras botas, las cuales podían aumentar a más de $1 \mathrm{~kg}$ en condiciones de clima húmedo, pasando por las utilizadas en el campeonato del mundo en 1954 de $380 \mathrm{~g}$, hasta las actuales de menos de $200 \mathrm{~g}^{2}$.

Concretamente las botas de fútbol, interfaz entre el cuerpo y el balón, se intentan mejorar, modificar y reinventar con la finalidad de intentar perfeccionar las habilidades de los futbolistas y prevenir lesiones ${ }^{2,3}$. Sus principales objetivos son proporcionar agarre a la superficie de juego, proteger el pie y facilitar el control y golpeo del balón².

Una de las principales iniciativas para mejorar estos parámetros fue la introducción de los tacos en forma de "cuchiIla" (alargados) para reemplazar el taco cónico tradicional en el diseño del sistema de fijación de las botas de fútbol para proporcionar, en teoría, mayor agarre y estabilidad ${ }^{2,3}$. Esta innovación causó controversia. Diferentes estudios indican que las botas con tacos alargados podrían predisponer a sufrir más lesiones que los tacos convencionales.

El diseño de la suela/tacos de las botas de fútbol varía en función de la amortiguación del terreno y están dirigidas a favorecer el gesto deportivo, proporcionando el agarre, la amortiguación y la tracción necesaria para cada superficie? ${ }^{2}$.

Los tipos de tacos que podemos encontrar en la actualidad son ${ }^{6}$ :

- Soft ground (SG); césped natural húmedo. LaS botas con suela/tacos SG están formadas con tacos de aluminio, o también podemos encontrarlos mixtos, que combinan el aluminio con material de goma.

Generalmente, la suela de las botas SG contienen de 6 a 8 tacos, y estos suelen ser largos y de forma redondeada. Tienen esta forma para proporcionar mayor penetración en el césped. El número de tacos es menor que en los otros tipos de botas para favorecer la penetración de estos en el terreno de juego, ya que la carga se distribuye en menos puntos de apoyo y, de este modo, se consigue una mayor tracción.

La amortiguación que proporciona el césped natural húmedo hace que no sea necesario repartir la carga en un mayor número de tacos; si el césped en vez de ser húmedo fuera seco, los tacos podrían molestar por tener menos amortiguación el terreno de juego ${ }^{6}$.

- Firm ground (FG); césped natural seco o artificial

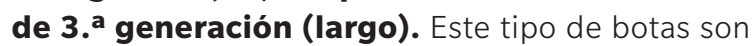
las más utilizadas por los jugadores de fútbol. Las botas con suela/tacos FG están formadas por tacos de goma o material similar, varían en forma y pueden ser redondeados o alargados.

El número de tacos es mayor que en las SG, ya que el terreno es más duro y necesita repartir las cargas en mayor superficie de apoyo. Generalmente presentan un mínimo de 12 tacos.

Son tacos relativamente largos, menos que los de las botas con suela/tacos SG. Esto se debe a que no penetran completamente en el terreno de juego (como sucede con las botas con suela/tacos SG) y a que la amortiguación del terreno es menor que en la de césped natural ${ }^{6}$.

- Artificial ground (AG) / mix ground (MG); césped artificial de 2. ${ }^{\text {a }}$ generación. Las botas con suela/tacos AG están compuestas por tacos de caucho redondos, generalmente, con diferentes longitudes, y varían en número según la forma de los tacos (de 13 a 22 tacos, aproximadamente). Esta composición está pensada para repartir la carga corporal en mayor puntos de apoyo y que no molesten los tacos a la hora de la práctica deportiva. Estos tacos permiten el agarre necesario para césped artificial de pelo corto (2. ${ }^{a}$ generación $)^{6}$.

- Turf o multitaco (TF); tierra, césped artificial de primera generación o moqueta. Las botas con suela/tacos TF están formadas por multitud de tacos de poca longitud, pueden variar en forma y número, se distribuyen en toda la totalidad de la suela de la bota de fútbol.

Este tipo de botas está pensada para la práctica deportiva en terrenos duros y abrasivos sin amortiguación alguna, como pueden ser los campos de tierra, los campos de césped artificial de primera generación (moqueta) $\mathrm{o}$, incluso, de cemento. Puesto que el terreno de juego no ofrece amortiguación, la distribución, el número y la longitud de los tacos son los que nos van a aportar esta amortiguación y la tracción necesaria para la práctica deportiva $^{6,7}$. 


\section{Relación de las lesiones en el futbol con las botas de fútbol}

En general, se ha hecho una distinción entre los denominados factores de riesgo intrínsecos (relacionados con la persona) y extrínsecos (relacionados con el medio ambiente). Los factores de riesgo intrínsecos están relacionados con las características biológicas o psicosociales individuales de una persona, como la edad, la inestabilidad articular, la fuerza muscular, la tensión muscular, la asimetría de la fuerza muscular, las lesiones anteriores, la adecuación de la rehabilitación y el estrés psicosocial. Los factores de riesgo extrínsecos se relacionan con variables ambientales como el nivel de juego, carga de ejercicio (cantidad de competencia y práctica), cantidad y nivel de entrenamiento, posición jugada, equipo (como espinilleras, cintas y zapatos), condiciones de campo de juego, reglas y juego sucio ${ }^{5,8}$.

Hay investigadores que creen que el tipo de calzado, así como la superficie de juego, tienen una influencia en los riesgos de lesiones en el fútbol. Parece obvio que las botas y las superficies que proporcionan una alta tracción durante las entradas y los movimientos de giro aumentarán el riesgo de esguinces de tobillo. Del mismo modo, se cree que una alta resistencia a la torsión de la parte delantera del pie durante movimientos de giro causará un mayor riesgo de lesiones de rodilla. Correr o realizar cambios repentinos en la dirección incrementa y al mismo tiempo influye en la dirección de las fuerzas que actúan sobre los miembros inferiores ${ }^{2}$.

Otros autores han investigado el diseño de botas de fútbol, tacos y los materiales utilizados en su construcción ${ }^{9-12}$. Durante la última década, el diseño de la bota de fútbol ha cambiado drásticamente. Los diseños modernos incorporan sistemas de sujeción o fijación (tacos) de tipo cónico con distintas longitudes y número en función de la zona anatómica del pie o alargados, e incluso una combinación de $\operatorname{ambos}^{2,13}$. Desde las primeras investigaciones que evalúan la bota de fútbol, se ha puesto de manifiesto la fijación del pie con la superficie de juego como una de las principales causas de las lesiones de tobillo y rodilla. Aunque los fabricantes indican que siguen un enfoque sistemático para decidir el diseño de la bota, existe una escasa investigación científica acerca de la influencia de los tacos y su relación con las lesiones ${ }^{2}$.

Los contactos de la superficie del zapato son importantes para el fútbol y, por lo tanto, la resistencia a la fricción debe mantenerse al mínimo. Las lesiones traumáticas, como los esguinces de rodilla, generalmente son causadas por la torsión de la rodilla que ocurre cuando el pie en un zapato con tacos atornillados se atasca en el suelo; dos tercios de las lesiones son causadas por uso excesivo de calzado de mala calidad. Otros autores también han sugerido que la zapatilla de fútbol puede ser un posible factor de riesgo de lesión ${ }^{8}$.

En consecuencia, este artículo tiene como objetivo principal identificar los distintos diseños de botas de fútbol y su relación con las lesiones del miembro inferior. El objetivo principal de este trabajo fue revisar qué evidencia hay sobre el diseño de botas de fútbol y la relación que tienen con las lesiones que se producen en los jugadores y con cambios cinéticos en el miembro inferior.

\section{MATERIAL Y MÉTODOS}

En esta revisión sistemática se ha realizado la búsqueda bibliográfica en bases de datos de PubMed, Scopus y Cumulative Index de Enfermería y Literatura Allied Health (CINAHL).

El procedimiento de esta revisión se llevó a cabo de acuerdo con un conjunto mínimo de elementos, basados en evidencias, para ayudar a presentar informes de revisiones sistemáticas y metanálisis (PRISMA). La búsqueda se ha realizado en las tres bases de datos siguiendo la misma estrategia y las mismas palabras clave: "soccer", "football", "injuries", "boots" y "shoes". La búsqueda se llevó a cabo entre los meses de enero y abril de 2020 .

Para el diseño de la estrategia de búsqueda la pregunta PICO fue usada por dos revisores. Pacientes: sujetos que jugaran a fútbol de cualquier edad. Intervención: ensayos clínicos aleatorizados y estudios clínicos en jugadores que usaban distinta tipología de botas donde fueron evaluados durante un periodo de seguimiento en las lesiones sufridas y cambios en la cinética del pie. Comparación: se compararon distintos diseños de botas, donde variaba el tipo de bota, el material, la suela y el tipo o longitud de tacos. Resultados: Los resultados obtenidos fueron a través de pruebas con distintas plataformas de presiones, plataforma de fuerzas, electromiografía, análisis de movimiento con VICON y registro de lesiones producidas durante la práctica deportiva.

La selección de los artículos la hicieron dos revisores cegados (T.R.M y E.L.R.). Los artículos fueron revisados de manera independiente observando el cumplimiento o no de los criterios de inclusión. En el caso de desacuerdo se resolvió mediante discusión entre ambos revisores.

Para la extracción de datos, se revisaron los títulos y resúmenes por dos revisores de manera independiente (T.R.M y E.L.R.). Se obtuvo el texto completo de los artículos que se consideraron relevantes. Los mismos revisores realizaron la lectura del texto y extrajeron los siguientes datos de cada estudio: detalles del estudio (autor y año de publicación), características de los participantes (edad media y sexo), diseño del estudio, tiempo de seguimiento e instrumento de medida.

Los artículos incluidos eran ensayos y estudios clínicos, cuyos criterios de inclusión y exclusión fueron los siguientes. Los criterios de inclusión que se han tenido en cuenta para la selección de artículos han incluido a la población general, sin centrarse en una edad específica, ni en género o profesionalidad a la hora de practicar fútbol, dado que es un deporte que se practica a nivel mundial, en todas las edades y categorías.

Se han excluido artículos que no cumplían con la metodología o rigor científico y estudios que no eran vinculantes a los objetivos marcados en este estudio, es decir, todos aqueIlos que no hablaran del calzado en el futbol o que no tuvieran 
una metodología aceptable. Para la evaluación de los artículos utilizamos la herramienta CASPe para análisis de ensayos clínicos (Critical Appraisal Skills Programme Español) ${ }^{14}$. Se seleccionaron los artículos cuyos resultados eran mayor o igual a 7 sobre 11 puntos; esta puntuación determina un nivel aceptable de evidencia según este método.

\section{RESULTADOS}

Inicialmente 241 estudios fueron identificados, pero 192 fueron eliminados tras estar duplicados al buscar en diferentes bases de datos. 131 estudios fueron eliminados por los criterios de inclusión y exclusión usando título, resumen y palabras clave. Por último, tras la lectura del texto completo, 26 artículos fueron eliminados por no cumplir con la metodología adecuada, quedándonos con 14 artículos que eran ensayos clínicos aleatorizados o estudios clínicos (Figura 1) (Tabla I).

\section{Características del estudio}

Esta revisión sistemática cuenta con un total de 17 artículos, en los cuales se incluyeron 760 sujetos, la edad media de los sujetos fue 19,5 años.

Los estudios clínicos contaron con un total de 158 sujetos, de los cuales 17 eran mujeres (10.75\%) y 141 varones (89.25\%). Los ensayos clínicos incluyeron 223 sujetos, de los cuales 14 fueron mujeres (6.75\%) y 209 varones (93.25\%).

En general todos los resultados muestran una relación multifactorial en la incidencia de lesiones en el fútbol. Las características de la bota, sobre todo la forma, número, longitud y localización de los tacos, puede ser un factor de riesgo para la lesión, aún más en combinación con factores intrínsecos, como la biomecánica, la forma física y anatomía del sujeto y factores extrínsecos, como la interacción de estos con la superficie de juego.

\section{DISCUSIÓN}

En los estudios seleccionados, hemos encontrado autores que estudiaron la incidencia de la forma de los tacos en las presiones plantares realizando gestos deportivos típicos del fútbol, como pueden ser los saltos, carrera recta, giros o golpeos de balón.

Los zapatos con menos tacos y más largos generaban una presión plantar significativamente mayor que los zapatos con un mayor número de tacos más cortos ${ }^{3,29}$.

Los tacos cónicos dieron valores más altos de presión plantar en la zona medial del pie y los tacos alargados mayor presión plantar en la zona lateral de medio pie y antepié. Las botas de tacos redondos pueden ser consideradas más seguras en función de la distribución de presión del pie y el patrón de centro de progresión de la presión imitaban el motivo nor-
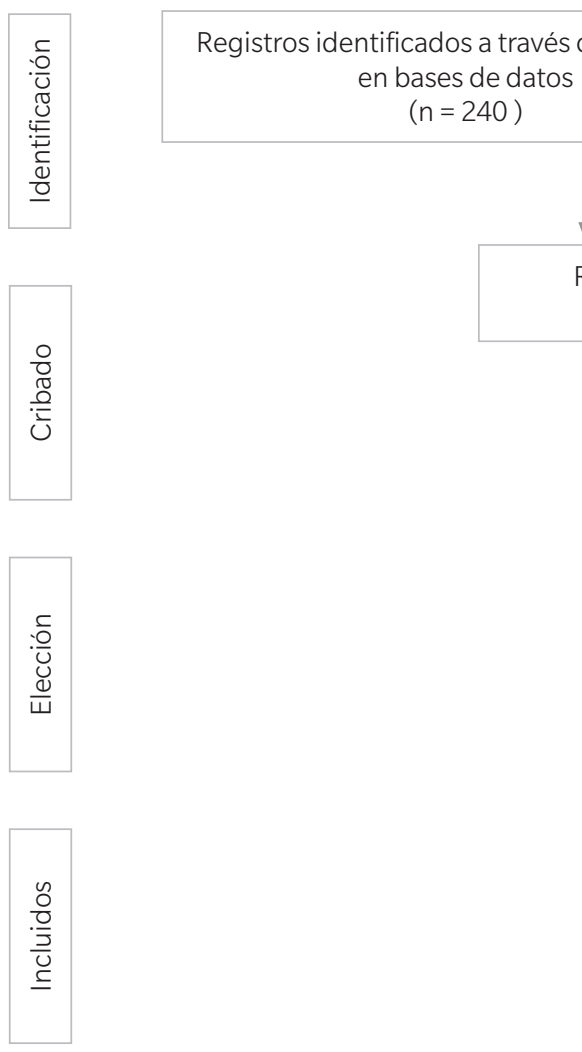

Registros después de eliminar los duplicados

Registros adicionales identificados a través de otras fuentes $(n=1)$

$$
(n=192)
$$
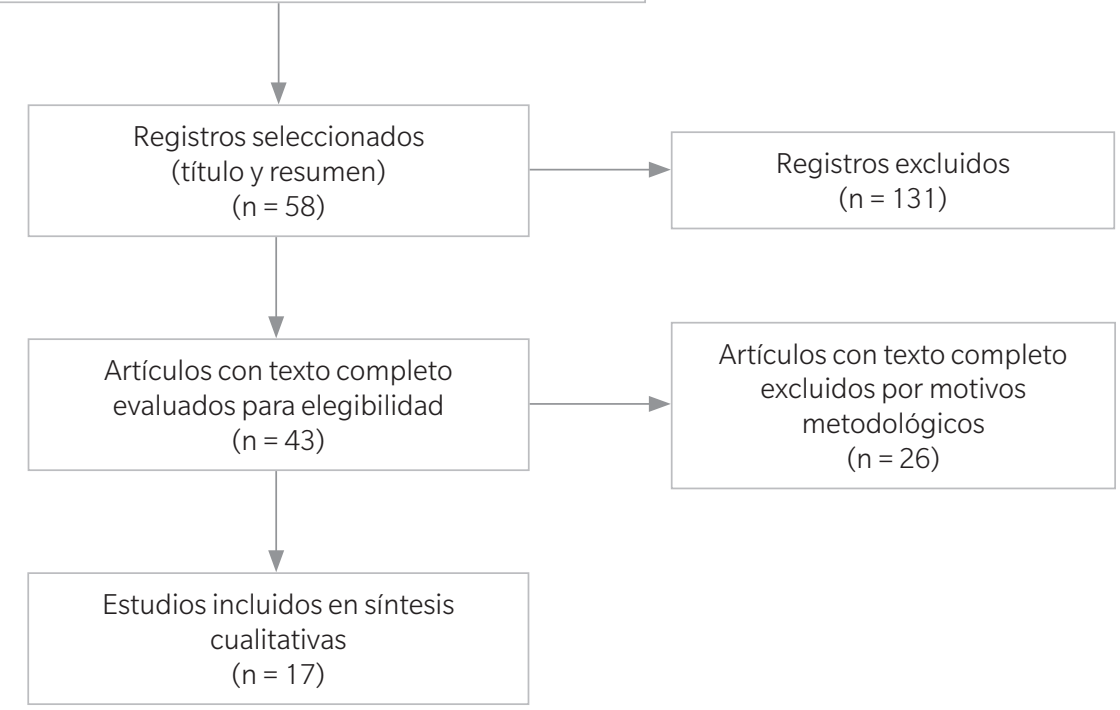

Figura 1. Diagrama de flujo. 
Tabla I. Resumen descriptivo de artículos seleccionados.

\begin{tabular}{|c|c|c|}
\hline Autor/es & Muestra & Método \\
\hline $\begin{array}{l}\text { Bentley y } \\
\text { cols. }^{15}\end{array}$ & 29 futbolistas & $\begin{array}{l}\text { Comparación de presiones plantares } \\
\text { en dos modelos diferentes de botas: } \\
\text { tacos cónicos y tacos alargados, en dos } \\
\text { pruebas diferentes: recta y slalom }\end{array}$ \\
\hline Kaila y cols. ${ }^{16}$ & $\begin{array}{l}15 \text { futbolistas } \\
\text { profesionales }\end{array}$ & $\begin{array}{l}\text { Estudio de la fuerza, en un espacio } \\
\text { tridimensional, a las que se somete } \\
\text { la tibia y la rodilla (valgo/varo/flexo- } \\
\text { extensión), en tres pruebas diferentes: } \\
\text { recta, cambios de sentido de } 30^{\circ} \text { y } 60^{\circ} \\
\text { con diferentes tipos de tacos }\end{array}$ \\
\hline
\end{tabular}

Okholm

Krygery cols. $^{17}$

8 futbolistas

\begin{tabular}{|c|c|}
\hline $\begin{array}{l}\text { Walter y } \\
\text { cols. }^{18}\end{array}$ & $\begin{array}{l}36 \text { futbolistas } \\
\text { (niños) }\end{array}$ \\
\hline
\end{tabular}

\begin{tabular}{ll}
\hline & \\
& \\
& \\
& \\
Azevedóvenes & adolescentes \\
cols. $^{19}$ & (edad: $14 \pm 0.7 /$ \\
& $14 \pm 0.5)$
\end{tabular}

\begin{tabular}{|c|c|c|}
\hline $\begin{array}{l}\text { Butler y } \\
\text { cols. }^{20}\end{array}$ & $\begin{array}{l}14 \text { jugadoras de } \\
\text { fútbol femenino } \\
\text { (edad: } 22.8 \pm \\
3.1 \text { años) y } 14 \\
\text { jugadores de fútbol } \\
\text { masculinos (edad: } \\
22.1 \pm 3.9 \text { años) }\end{array}$ & $\begin{array}{l}\text { Se sometieron a una evaluación de } \\
\text { análisis de movimiento mientras } \\
\text { realizaban un de salto de cabeza. Cada } \\
\text { sujeto realizó la tarea en tres condiciones } \\
\text { diferentes de calzado (calzado para } \\
\text { correr, taco alargados y multitacos). Se } \\
\text { utilizaron análisis de varianza } \\
\text { bidireccionales para examinar las } \\
\text { diferencias estadísticas en la mecánica } \\
\text { de aterrizaje entre las condiciones del } \\
\text { calzado mientras se controlaban las } \\
\text { diferencias de género }\end{array}$ \\
\hline
\end{tabular}

Comparación de la comodidad subjetiva del sujeto con las presiones plantares obtenidas, usando tres tipos diferentes de tacos Firm Ground

Análisis de la postura del pie durante la carrera en niños con botas de tacos en comparación con calzado deportivo, para evaluar la influencia de las botas de tacos sobre la apofisitis calcánea en niños

Se dividió en un grupo de jugadores de fútbol $(n=15)$ y un grupo de control $(n=15)$. La presión plantar media se determinó para siete diferentes regiones del pie. Los datos se compararon entre el pie preferido y el no preferido, y entre los grupos, con el pie descalzo en una plataforma de presiones

Se sometieron a una evaluación de análisis de movimiento mientras realizaban un de salto de cabeza. Cada diferentes de calzado (calzado para correr, taco alargados y multitacos). Se utilizaron análisis de varianza bidireccionales para examinar las de aterrizaje entre las condiciones del diferencias de género

\section{Resultados}

En la recta la PP resultó mayor en la parte lateral del pie y en el slalom la PP fue mayor en la zona medial del talón. Los tacos cónicos dieron valores más altos de la PP en la zona medial del pie y los tacos alargados mayor PP en la zona lateral de medio pie y antepié

Se detectó un aumento de los momentos de valgo y rotación interna de la tibia en los cambios de sentido con una flexión menor a $30^{\circ}$ en la articulación de la rodilla. Se recomienda unos tacos tipo Soft-Ground, más largos y de metal, para césped natural y tacos aplanados más cortos para césped artificial, para una adecuada penetración y tracción

El confort (subjetivo) no es una medida fiable para el diseño de unas botas de fútbol; la medida de presión plantar es necesaria para optimizar la prevención de lesiones en el diseño de las botas de tacos

La mayoría de los sujetos de este estudio resultaron mantener una dorsiflexión aumentada durante las fases de la carrera con las botas de tacos, lo que aumentaba la carga del talón considerablemente

Jóvenes futbolistas presentan asimetrías en la presión plantar en el hallux, $5 .^{\circ}$ metatarsiano y parte posterior media del pie, con mayor presión observada en el no pie preferido. Las asimetrías observadas en los jóvenes futbolistas sugieren que tienen adaptación específica que puede resultar de demandas mecánicas durante la práctica de fútbol

Los resultados del estudio actual sugieren que la mecánica de aterrizaje se ve afectada de manera diferente por las variaciones en el calzado y el género. Además, se observaron diferencias consistentes en la mecánica de aterrizaje entre los diferentes tipos de calzado y entre los géneros. Se observaron interacciones significativas para el género y el calzado en el tobillo y la rodilla para el segundo aterrizaje. En el calzado más rígido, los sujetos femeninos exhibieron un movimiento reducido en el tobillo y la rodilla al aterrizar después de una tarea de salto en comparación con los sujetos masculinos. Los jugadores de fútbol masculinos exhibieron un aumento de grado en la flexión dorsal en los tacos alargados

En el mediopié, los valores pico de presión aumentaron significativamente en botas de fútbol en comparación con zapatillas para correr debajo del lateral mediopié a $149 \%$ (pie preferido) y $140 \%$ (no preferido pie) pero no debajo del mediopié medio. Las cabezas metatarsianas demostraron un aumento estadísticamente significativo del pico presión plantar en las botas de fútbol en comparación con las zapatillas para correr de hasta el $139 \%$, con la excepción de los no preferidos cabezas metatarsianas 2 y 3 
Tabla I (Cont.). Resumen descriptivo de artículos seleccionados.

\begin{tabular}{|c|c|c|}
\hline Autor/es & Muestra & Método \\
\hline Elis y cols..$^{9}$ & $\begin{array}{l}21 \text { jugadores } \\
\text { de fútbol } \\
\text { masculino } \\
(25.5 \pm 1,8 \\
\text { años) }\end{array}$ & $\begin{array}{l}\text { El sistema Pedar Mobile se utilizó para } \\
\text { recopilar información sobre la presión } \\
\text { plantar dentro del zapato de fútbol. Se } \\
\text { realizaron cuatro movimientos específicos } \\
\text { de fútbol (carrera normal, maniobra de } \\
\text { corte, sprint y tiro al arco) }\end{array}$ \\
\hline $\begin{array}{l}\text { Gehring } \\
\text { y cols. }{ }^{22}\end{array}$ & $\begin{array}{l}6 \text { jugadores } \\
\text { de fútbol } \\
\text { masculino } \\
\text { (25.2 [ } \pm 1.4] \\
\text { años) }\end{array}$ & $\begin{array}{l}\text { Los sujetos realizaron movimientos de } \\
\text { giro complejos con zapatos de fútbol } \\
\text { con tacos alargados y redondos. Se } \\
\text { registraron las fuerzas de reacción del } \\
\text { terreno, la cinemática 3D y la actividad } \\
\text { electromiog ráfica de los músculos de la } \\
\text { parte inferior de la pierna }\end{array}$ \\
\hline $\begin{array}{l}\text { Nuns y } \\
\text { cols. }{ }^{23}\end{array}$ & $\begin{array}{l}\text { 9 jugadores } \\
\text { de fútbol } \\
\text { (edad } 15.7 \pm \\
1.6 \text { años) }\end{array}$ & $\begin{array}{l}\text { Este estudio investigó el efecto de la } \\
\text { configuración de los tacos y los niveles de } \\
\text { amortiguación de la plantilla en percepción } \\
\text { de comodidad y presiones plantares en el } \\
\text { zapato en el talón y la cabeza del quinto } \\
\text { metatarsiano. Usaron dos modelos de } \\
\text { botas y dos plantillas diferentes. ( } 6 \text { y } 8 \\
\text { tacos, gel y porón/ gel). Realizaron pruebas } \\
\text { de carrera y giro de } 180^{\circ}\end{array}$ \\
\hline
\end{tabular}

\begin{tabular}{|c|c|c|}
\hline $\begin{array}{l}\text { Olivera y } \\
\text { cols. }{ }^{24}\end{array}$ & $\begin{array}{l}10 \text { jugadores } \\
\text { de fútbol }\end{array}$ & $\begin{array}{l}\text { Los datos cinemáticos fueron recolectados } \\
\text { usando el sistema Vicon y una plataforma } \\
\text { de fuerza AMTI. El sistema Nexus calculó } \\
\text { los momentos y los picos se extrajeron en } \\
\text { Matlab en cada } 10 \% \text { de la fase de apoyo }\end{array}$ \\
\hline
\end{tabular}

\section{Resultados}

Los resultados mostraron patrones de distribución de presión característicos con áreas de carga específicas del pie que corresponden a los movimientos evaluados. Además, se encontraron patrones de carga con valores de presión más altos que los observados durante el funcionamiento normal. En el corte, la parte medial del pie; en sprint, el primer y segundo radio; $y$ al patear, la parte lateral del pie está predominantemente cargada. No se encontró ningún efecto global de las dos superficies en los parámetros de presión

Los momentos calculados de la articulación externa de la rodilla fueron similares con ambas configuraciones de tacos, aunque hubo una tendencia hacia un aumento vertical y anterior-posterior de las fuerzas de reacción del suelo con tacos alargados. La electromiografía evidenció una activación significativamente mayor del cuádriceps femoral con tacos redondos durante la fase inicial de la postura

La plantilla de Poron generalmente proporciona menor presiones máximas que la plantilla de Poron/gel, particularmente durante el paso de frenado del giro. El modelo de tacos no influyó independientemente en las presiones máximas en el quinto metatarsiano, y tuvo influencia mínima en las cargas del talón. La bota de 8 tacos y la plantilla de Poron se desempeñaron mejor biomecánicamente y perceptualmente, pero la condición combinada no lo hizo

Los tacos circulares mostraron un momento de rotación externa mayor al inicio de la fase de apoyo que los triangulares y los mixtos. En el movimiento de patada, los mixtos mostraron un momento valgo mayor que los triangulares y redondos en el primer $10 \%$ de la postura. Los mixtos también obtuvieron un mayor momento de rotación interna al $60 \%$ de la postura. Estos resultados sugieren que los redondos pueden ofrecer una mayor estabilidad al inicio de la fase de apoyo

Existieron diferencias significativas de género en la fuerza y el tiempo de fuerza integral debajo del mediopié lateral y el antepié durante la tarea de corte cruzado, así como en el antepié medio durante la tarea de corte lateral con los hombres demostrando una mayor fuerza. No existieron diferencias significativas en la carga en el lado medial del pie durante ninguna tarea

Los resultados de este estudio indican que el aumento de la carga plantar en la porción lateral del mediopié y el antepié en los hombres podría ser una posible explicación de la mayor incidencia de fracturas por estrés del quinto metatarsiano en los hombres

Se compararon dos diseños de tacos redondos tradicionales y un diseño de tacos alargados con una zapatilla de running estándar como condición

12 jugadores de fútbol Stefanyshyn masculino y cols. ${ }^{26}$ $(26.4 \pm 6.2$ años) de control. La tracción traslacional y rotacional de los zapatos en césped artificial se midió utilizando una plataforma Stewart. Los momentos de la articulación del tobillo y la rodilla se cuantificaron mientras realizaban movimientos de corte y giro a $4.0 \mathrm{~m} / \mathrm{s}$ en el mismo césped artificial
Todos los zapatos de fútbol tuvieron una tracción rotacional significativamente mayor que los zapatos para correr, pero no hubo diferencias significativas entre los diseños de tacos. El diseño de los tacos alargados tenía una tracción traslacional significativamente mayor que los diseños de tacos tradicionales. Como resultado, el diseño de tacos que fue probado parece efectivo para desacoplar las características de tracción traslacional y rotacional de los zapatos de fútbol, lo que podría ser beneficioso para reducir las lesiones. No hubo diferencias significativas en los momentos resultantes de la articulación del tobillo y la rodilla entre los zapatos durante el movimiento de corte 
Tabla I (Cont.). Resumen descriptivo de artículos seleccionados.

\begin{tabular}{|c|c|c|c|}
\hline Autor/es & Muestra & Método & Resultados \\
\hline Wong y cols. ${ }^{27}$ & $\begin{array}{l}15 \text { jugadores } \\
\text { de fútbol } \\
\text { masculinos } \\
\text { (edad } 20.9 \pm \\
1.3 \text { años) }\end{array}$ & $\begin{array}{l}\text { La presión plantar se registró en cinco } \\
\text { pruebas exitosas en cada uno de los } \\
\text { cuatro movimientos relacionados con el } \\
\text { fútbol: correr, cortar de lado, } 45^{\circ} \text { corte y } \\
\text { aterrizaje desde un salto vertical. Cada } \\
\text { huella se dividió en } 10 \text { áreas registradas } \\
\text { para su análisis }\end{array}$ & $\begin{array}{l}\text { El presente estudio mostró que los cuatro movimientos } \\
\text { relacionados con el fútbol tuvieron una influencia } \\
\text { significativa en la amplitud y distribución de la presión. En } \\
\text { general, corte lateral y } 45^{\circ} \text { el corte tenía una presión más } \\
\text { alta, mientras que el aterrizaje en salto tenía una presión } \\
\text { más baja en comparación con la carrera. Entre estos } \\
\text { movimientos se encontraron presiones más altas en el } \\
\text { lado medial de la superficie plantar. En particular, el hallux, } \\
\text { el antepié medial, el antepié central y el talón medial } \\
\text { recibieron presiones más altas, lo que sugiere que estas } \\
\text { áreas pueden tener mayores riesgos de lesiones }\end{array}$ \\
\hline Wong y cols. ${ }^{28}$ & $\begin{array}{l}15 \text { jugadores } \\
\text { universitarios } \\
\text { masculinos } \\
\text { (edad } 20.9 \pm \\
1.3 \text { años) }\end{array}$ & $\begin{array}{l}\text { Para registrar la distribución de la } \\
\text { presión plantar, los jugadores usaron } \\
\text { aleatoriamente } 3 \text { tipos de zapatos de } \\
\text { fútbol (clásico de } 6 \text { y } 12 \text { espigas, y } 12 \\
\text { espigas especialmente diseñados) } \\
\text { incrustados con un dispositivo } \\
\text { registrador de presión de la plantilla con } \\
99 \text { sensores, divididos en } 10 \text { áreas para } \\
\text { el análisis. La presión plantar se registró } \\
\text { en } 5 \text { pruebas exitosas en cada uno de los } \\
\text { cuatro movimientos relacionados con el } \\
\text { fútbol: correr (a } 3.3 \mathrm{~m} / \mathrm{s} \text { ), corte lateral, } \\
45^{\circ} \text { corte y aterrizaje desde un salto } \\
\text { vertical }\end{array}$ & $\begin{array}{l}\text { El presente estudio indica que la presión plantar en } \\
\text { el pie preferido y no preferido no es simétrica. En } \\
\text { general, se encuentra una presión plantar más alta en } \\
\text { el pie preferido, en los tres tipos de zapatos y cuatro } \\
\text { movimientos investigados. Por lo tanto, el pie preferido } \\
\text { y no preferido debe considerarse por separado en } \\
\text { futuros estudios, así como en los programas de ejercicios } \\
\text { que tienen como objetivo la prevención de lesiones. } \\
\text { Además, debido a la asimetría entre el pie preferido y el } \\
\text { no preferido, se sugiere que se use un inserto más duro } \\
\text { para el pie no preferido para mejorar la estabilidad, y un } \\
\text { inserto más suave para el pie preferido para absorber/ } \\
\text { reducir la presión plantar }\end{array}$ \\
\hline Dong y cols. ${ }^{29}$ & $\begin{array}{l}14 \text { jugadores } \\
\text { de fútbol } \\
\text { masculino } \\
\text { (edad, } 19.7 \pm \\
1.2 \text { años) }\end{array}$ & $\begin{array}{l}\text { Los participantes completaron tareas } \\
\text { de carrera recta y corte lateral de } 45^{\circ} \\
\text { a la izquierda, respectivamente, a una } \\
\text { velocidad de } 5.0 \pm 0.2 \mathrm{~m} / \mathrm{s} \text { en césped } \\
\text { natural. Seleccionaron zapatos de fútbol } \\
\text { con diseño de terreno firme (FG), diseño } \\
\text { de terreno artificial (AG) y tacos de } \\
\text { césped (TF) al azar }\end{array}$ & $\begin{array}{l}\text { La FG mostró una fuerza de reacción del suelo horizontal } \\
\text { (GRF) significativamente mayor y una relación de tracción } \\
\text { promedio requerida en comparación con AG y TF. FG } \\
\text { mejoraría el rendimiento atlético en el césped natural, pero } \\
\text { también puede asumir mayores riesgos de lesiones sin } \\
\text { contacto en comparación con AG y TF }\end{array}$ \\
\hline Silva y cols. ${ }^{30}$ & $\begin{array}{l}82 \text { jugadores } \\
\text { de fútbol } \\
\text { masculino } \\
\text { (edad } 18-30 \\
\text { años) }\end{array}$ & $\begin{array}{l}\text { Jugadores con y sin inestabilidad crónica } \\
\text { de tobillo. Todos los sujetos realizaron } 2 \\
\text { series de } 6 \text { saltos cruzados consecutivos } \\
\text { con pie dominante, cada uno con uno } \\
\text { de los cuatro modelos de tacos (TF, AG, } \\
\text { HG, FG) }\end{array}$ & $\begin{array}{l}\text { No se identificaron efectos e interacciones principales } \\
\text { de tacos y grupos estadísticamente significativos en la } \\
\text { magnitud cinemática, cinética y electromiográfica de los } \\
\text { músculos peroneos. Se observó un efecto principal del } \\
\text { grupo para el tiempo de activación del peroneo largo para } \\
\text { el modelo }\end{array}$ \\
\hline
\end{tabular}

mal, mientras que las botas de tacos alargados podrían considerarse potencialmente de forma relativa más perjudiciales debido a la mayor carga no natural en la mitad lateral del pie, lo que podría predisponer a las lesiones ${ }^{15}$. La presión máxima en el antepié lateral (región de $5^{\circ}$ metatarsiano) en los tacos alargados fue mayor que en los triangulares y redondos en los movimientos de corte. La presión máxima y la fuerza-tiempo integral en el antepié medial (1 radio) y central (2-4 metatarsianos) en los tacos triangulares fueron más pequeños que los tacos redondos. El taco alargado tiene un mayor riesgo de lesión ósea del quinto metatarsiano. En estos artículos, Benteley y cols. ${ }^{15}$ detectan las botas con tacos alargados como un riesgo de lesión en la parte lateral del pie, ya que los resultados muestran que estas botas generan picos mayores de presión plantar en la parte lateral del pie.
Por otra parte, Nuns y cols. ${ }^{23}$ evaluaron los valores de presiones plantares en la zona del talón y el quinto metatarsiano en botas de 6 y 8 tacos, combinándolas con unas plantillas de porón y otras de porón/gel. Resultó generar menores presiones las plantillas de porón frente a las de porón/gel y la bota de 8 tacos frente a la de 6.

Por otro lado, Wong y cols. ${ }^{27}$ y Henning y cols. ${ }^{5}$ hablan de las presiones plantares que generan las botas de tacos redondos en la parte medial del pie, y la capacidad de estas para generar lesiones en la parte medial del pie. Se encontraron presiones más altas en el lado medial de la superficie plantar en botas de tacos redondos. En particular, el hallux, el antepié medial, el antepié central y el talón medial recibieron presiones más altas, lo que sugiere que estas áreas pueden tener mayores riesgos de lesiones ${ }^{27}$. Carl y cols. ${ }^{21}$ en su estudio, también 
encontraron en el mediopié que los valores pico de presión aumentaron significativamente en botas de fútbol en comparación con zapatillas para correr debajo del lateral mediopié. Con respecto a los dedos de los pies, los valores pico de presión aumentaron significativamente en botas de fútbol en comparación con zapatillas para el dedo gordo del pie ${ }^{21}$.

Azevedo y cols. ${ }^{19}$ refieren que encontraron en su estudio picos de presiones más altos en niños futbolistas en 1. a y $5 .{ }^{\text {a }}$ cabeza metatarsal de los pies no preferidos. Las asimetrías observadas en los jóvenes futbolistas sugieren que tienen adaptación específica que puede resultar de demandas mecánicas durante la práctica de fútbol ${ }^{19,28}$.

Apoyando los resultados del estudio anterior, Carl y cols. ${ }^{21}$ en su estudio, donde comparan las presiones plantares usando botas fútbol con el uso de calzado deportivo, encuentran que las cabezas metatarsianas del pie preferido mostraron un aumento estadísticamente significativo del pico presión plantar en las botas de fútbol en comparación con las zapatillas para correr, con la excepción de los pies no preferidos que se daban en las cabezas metatarsianas 2. ${ }^{a}$ y 3. ${ }^{\text {a21 }}$.

Por otra parte, tenemos estudios donde los autores se centraron en investigar la capacidad de tracción y fricción de los diferentes tipos de tacos con diferentes superficies de juego. El diseño y la disposición de los tacos son características importantes del zapato que permiten aceleraciones y paradas rápidas, cortes rápidos y giros ${ }^{5}$. En superficies duras, se puede inferir que aumentar el número de tacos mejora la distribución del estrés, y la altura del taco debe limitarse para maximizar la penetración y permitir un estrés mejor distribuido a lo largo de la suela exterior. Sin embargo, aumentar el número y la distribución de los tacos debe evaluarse cuidadosamente para evitar un diseño que pueda resistir fuertemente las rotaciones axiales ${ }^{13}$.

Blachard y cols. ${ }^{13}$ en su revisión concluyen que el comportamiento de los tacos puede ser sensible a la rigidez del suelo: los tacos no se hunden completamente en una superficie dura y el contacto no ocurre en toda la suela. En este contexto, se observan cambios en las propiedades de tracción con un mayor riesgo de lesiones y deterioro del rendimiento ${ }^{13}$. Poniendo otra vez de manifiesto, lo citado anteriormente, la longitud y número de tacos debe variar en función del terreno de juego.

En un análisis de la influencia de la forma de los tacos FG en las articulación de la rodilla, Kaila y cols. ${ }^{16}$ detectaron un aumento de los momentos de valgo y rotación interna de la tibia en los cambios de sentido con una flexión menor a $30^{\circ}$ en la articulación de la rodilla.

En el estudio de Olivera y cols. los tacos circulares mostraron un momento de rotación externa mayor al inicio de la fase de apoyo que los triangulares y los mixtos. En el movimiento de patada, los mixtos mostraron un momento valgo mayor que los triangulares y redondos en el primer $10 \%$ de la postura. Los mixtos también obtuvieron un mayor momento de rotación interna al $60 \%$ de la postura. Estos resultados sugieren que los redondos pueden ofrecer una mayor estabilidad al inicio de la fase de apoyo. Los tacos de fútbol influ- yen en el momento de la articulación de la rodilla durante los movimientos de corte y patadas ${ }^{24}$.

\section{CONCLUSIÓN}

Dado que los resultados de los estudios han mostrado tener en común en la incidencia de lesiones en el fútbol un patrón multifactorial, es difícil controlar y determinar con seguridad todos los factores que entran en juego en el origen de la lesión.

En cuanto al diseño de la bota, se puede decir que la configuración de los tacos, en cuanto a número, forma y longitud, está indicada para diferentes terrenos de juego, teniendo en cuenta la capacidad de penetración y amortiguación necesaria para cada superficie.

Superficies blandas: menor número de tacos y más largos, en cuanto a la forma serían mejor los redondos. Tipo Soft Ground (SG) (césped natural húmedo). Superficies duras: un número mayor de tacos que en el caso de césped húmedo y menor longitud, pudiendo variar la forma. Tipo Firm Groun (FG) (césped natural seco y césped artificial de última generación). Superficies más duras: muchos tacos distribuidos por la totalidad de la suela, con poca longitud. Tipo Artificial ground (AG) o Turf (TF) (césped artificial de 2. ${ }^{a}$ generación en el caso de las AG o césped artificial de $1 .^{a}$ generación, moqueta o tierra).

Analizando las lesiones más comunes en el fútbol y relacionándolas con el calzado:

- Las fracturas de estrés de $5 .^{\circ}$ metatarsiano (más común en adultos varones) están más relacionadas con taco alargado y pueden tener cierta relación con factores intrínsecos, como la biomecánica.

- La lesión del LCA (más frecuente en mujeres que en hombres) puede estar más relacionada con tacos más largos, redondos y superficies secas, con mayor capacidad de penetración, además de la biomecánica, fuerza muscular y anatomía.

- Los esguinces o inestabilidad de tobillo no están relacionados con la forma, número o longitud del taco, sino que las botas actuales son de corte bajo y no protegen lo suficiente al tobillo de las torsiones, además de estar influenciado por la biomecánica, anatomía y fuerza muscular.

- La apofisitis calcánea en niño puede estar relacionada a la poca amortiguación del calzado (por una elección de tacos errónea en relación al terreno) que hace que se mantenga la flexión dorsal del pie en la carrera y esto genere mayores "fuerzas compresivas" en el talón y estrés tensil del sistema aquíleo calcáneo plantar.

- Los trastornos del primer radio pueden estar más relacionadas a los tacos redondos, la biomecánica y la rigidez de la bota en general, la zona de la articulación metatarsofalángica debe ofrecer cierta flexibilidad y la zona del mediopié evitarla. 


\section{CONFLICTO DE INTERESES}

Ninguno de los autores presenta conflicto de intereses relacionado con la realización del presente artículo

\section{FINANCIACIÓN}

El presente artículo no ha tenido ningún tipo de financiación.

\section{BIBLIOGRAFÍA}

1. Real Federación de fútbol. Informe de licencias tras el cierre de temporada 2015/2017 [Internet]. Medio Oficial Real Federación Española de Fútbol. 2017. Disponible en: https://www.rfef.es/transparencia/licencias

2. Mosqueira M. Las botas de fútbol y los distintos sistemas de fijación: diseño, presiones plantares y su relación con las lesiones del miembro inferior. Revista de Preparación Física en el Fútbol. 2014;02/01/2014:922. Disponible en: https://futbolpf.org/wp-content/uploads/2018/01/ Revista-11.pdf

3. Hilgers MP, Walther M. Evolution of Soccer Shoe Design. Int J Athl Ther Train. 2011;16(3):1-4. DOI: 10.1123/ijatt.16.3.1.

4. Olaso Melis J, Unanue García A, Navarro García J, Gámez Payá J, Ferrandis Ferrer R, Medina Ripoll E, et al. Diseño innovador de botas de fútbol para hierba artificial. Revista de Biomecánica. 2011; 6:35-40.

5. Hennig EM. The influence of soccer shoe design on player performance and injuries. Res Sports Med Print. 2011;19(3):186-201. DOI: 10.1080/15438627.2011.582823.

6. Tipos de tacos en las botas de fútbol. [Internet]. Decathlon. 2020 [citado 9 de marzo de 2020]. Disponible en: https://comunidad.decathlon.es/futbol/opiniones-sobre/tipos-tacos-botas-futbol/5e63a153d5b55720e5f09ab8

7. Mansfield MM, Bucinell RB. Effects of playing surface and shoe type on ACL tears in soccer players. Am J Eng Appl Sci. 2016;9(4):1150-7. DOI: 10.3844/ajeassp.2016.1150.1157.

8. Dvorak J, Junge A. Football Injuries and Physical Symptoms. A review of the literature. Am J Sports Med. 2000;28(5 Suppl):S3-9. DOI: 10.1177/28.suppl_5.s-3.

9. Eils E, Streyl M, Linnenbecker S, Thorwesten L, Völker K, Rosenbaum D. Characteristic plantar pressure distribution patterns during soccer-specific movements. Am J Sports Med. 2004;32(1):140-5. DOI: 10.1177/0363546503258932.

10. Garrick JG, Requa RK. Football cleat design and its effect on anterior cruciate ligament injuries. Am J Sports Med. 1996;24(5):705-6. DOI: 10.1177/036354659602400529

11. Livesay GA, Reda DR, Nauman EA. Peak torque and rotational stiffness developed at the shoe-surface interface: the effect of shoe type and playing surface. Am J Sports Med. 2006;34(3):415-22. DOI: 10.1177/0363546505284182.

12. Majid F, Bader DL. A biomechanical analysis of the plantar surface of soccer shoes. Proc Inst Mech Eng H. 1993;207(2):93-101. DOI: 10.1243/PIME_PROC_1993_207_276_02.

13. Blanchard S, Palestri J, Guer JL, Behr M. Current Soccer Footwear, Its Role in Injuries and Potential for Improvement. Sports Med Int Open. 2018;02(2):E52-61. DOI: 10.1055/a-0608-4229.
14. Cabello LópezJB. Lectura crítica de la evidencia clínica. Barcelona: Elsevier España; 2015.

15. Bentley JA, Ramanathan AK, Arnold GP, Wang W, Abboud RJ. Harmful cleats of football boots: a biomechanical evaluation. Foot Ankle Surg OffJ Eur Soc Foot Ankle Surg. 2011;17(3):140-4. DOI: 10.1016/j. fas.2010.04.001.

16. Kaila R. Influence of modern studded and bladed soccer boots and sidestep cutting on knee loading during match play conditions. Am J Sports Med. 2007;35(9):1528-36. DOI: 10.1177/0363546507300257.

17. Okholm Kryger K, Jarratt V, Mitchell S, Forrester S. Can subjective comfort be used as a measure of plantar pressure in football boots? J Sports Sci. 2017;35(10):953-9. DOI: 10.1080/02640414.2016.1206661.

18. Walter JH, Ng GK. The evaluation of cleated shoes with the adolescent athlete in soccer. The Foot. 2002;12(3):158-65. DOI: 10.1054/ foot.2002.0729

19. Azevedo RR, da Rocha ES, Franco PS, Carpes FP. Plantar pressure asymmetry and risk of stress injuries in the foot of young soccer players. Phys Ther Sport. 2017;24:39-43. DOI: 10.1016/j.ptsp.2016.10.001.

20. Butler RJ, Russell ME, Queen R. Effect of soccer footwear on landing mechanics. Scand J Med Sci Sports. 2014;24(1):129-35. DOI: 10.1111/j.1600-0838.2012.01468.x.

21. Carl H-D, Pauser J, Swoboda B, Jendrissek A, Brem M. Soccer boots elevate plantar pressures in elite male soccer professionals. Clin J Sport Med. 2014;24(1):58-61. DOI: 10.1097/01.jsm.0000432857.79305.6c.

22. Gehrind D, Rott F, Stapelfeldt B, Gollhofer A. Effect of soccer shoe cleats on knee joint loads. Int J Sports Med. 2007;28(12):1030-4. DOI: 10.1055/s-2007-965000.

23. Nunns MPI, Dixon SJ, Clarke J, Carré M. Boot-insole effects on comfort and plantar loading at the heel and fifth metatarsal during running and turning in soccer. J Sports Sci. 2016;34(8):730-7. DOI: 10.1080/02640414.2015.1069378.

24. Rosa de Olivera W, Onodera AN, La Torre M. Influence of cleat design on knee joint moments during cutting maneuver and instep kick in soccer. Muscles Ligaments Tendons J. 2019;9(3):386-94. DOI: 10.32098/ mltj.03.2019.13

25. Sims EL, Hardaker WM, Queen RM. Gender differences in plantar loading during three soccer-specific tasks. Br J Sports Med. 2008;42(4):2727. DOI: $10.1136 /$ bjsm.2007.042432.

26. Stefanyshyn DJ, Lee J, Park S. The influence of soccer cleat design on resultant joint moments. Footwear Sci. 2010;2(1):13-9. DOI: 10.1080/19424280903535454.

27. Wong PL, Chamari K, Mao de W, WisløffU, Hong Y, Wong P, et al. Higher plantar pressure on the medial side in four soccer-related movements. Br J Sports Med. 2007;41(2):93-100. DOI: 10.1136/bjsm.2006.030668.

28. Wong PL, Chamari K, Chaouachi A, Mao de W, Wisløff U, Hong Y, et al. Difference in plantar pressure between the preferred and non-preferred feet in four soccer-related movements. Br J Sports Med. 2007;41(2):8492. DOI: 10.1136/bjsm.2006.030908.

29. Dong S, Gu Y, Mei Q, Baker J. Different soccer stud configurations effect on running and cutting movements. Int J Biomed Eng Technol. 2017;24:19. DOI: 10.1504/IJBET.2017.083814

30. Silva DCF, Macedo R, Montes AM, Santos R, Vilas-Boas JP, Sousa ASP. Does the cleat model interfere with ankle sprain risk factors in artificial grass? Clin Biomech. 2019;63:119-26. DOI: 10.1016/j.clinbiomech.2019.03.004 